\title{
Implicit Sentiment Identification using Aspect based Opinion Mining
}

\author{
Sayali A Mankar ${ }^{1}$, M. D. Ingle ${ }^{2}$ \\ ${ }^{1,2}$ Department of Computer Engineering, Jaywantrao Sawant College of Engineering, Hadapsar \\ ${ }^{2}$ Professor, Department of Computer Engineering, Jaywantrao Sawant College of Engineering, Hadapsar
}

\begin{abstract}
Opinion mining or sentiment analysis is the computational study of opinions or emotions towards aspects or things. The aspects are nothing but attributes or components of the individuals, events, topics, products and organizations. Opinion mining has been an active research area in Web mining and Natural Language Processing (NLP) in recent years. With the explosive growth of $E$ commerce, there are millions of product options available and people tend to review the viewpoint of others before buying a product. An aspect-based opinion mining approach helps in analyzing opinions about product features and attributes. This project is based on extracting aspects and related customer sentiments on tourism domain. This offers an approach to discover consumer preferences about tourism products and services using statistical opinion mining. The proposed system tries to extract both explicit aspects as well as implicit aspects from customer reviews. It thus increases the sentiment orientation of opinion. Most of the researches were based on explicit opinions of customers. This system tries to retrieve implicit sentiments. Due to the growing availability of unstructured reviews, the proposed system gives a summarized form of the information that is obtained from the reviews in order to furnish customers with pin point or crisp results.
\end{abstract}

Keywords: opinion mining, implicit, explicit, customer preferences

\section{Introduction}

What other people think has always been an important part of our information gathering. With the ease of availability and growing popularity of opinion-rich resources such as online review sites and personal blogs, there arise millions of opportunities and challenges as people now actively use information technologies to find out and analyze the opinions of others [1].

Opinions or sentiments consists of thwarted expressions, irony and may contain ambiguous or even implicit sentiments. Traditional information extraction techniques are often developed for formal genre (e.g. news, scientific papers), which cannot apply effectively for opinion mining or sentiment analysis. The project aims to automatically extract fine-grained information from opinion documents. Their data sources are often ungrammatical and noisy, containing spelling errors (e.g. improper capitalization), abbreviations, slang and emotions. Aspect extraction remains to be a challenging problem for opinion mining or sentiment analysis. On the other hand, they are critically important, because without knowing aspects and entities in a corpus, the mined opinions have little use.

Challenges to be addressed are Identification of aspects (Explicit as well as implicit) related to customer opinions. Polarity conflicting issue of document level aspect-based anal-ysis will be resolved by using sentence level aspect based analysis, aspect-based segmentation will be done wherein a multi aspect review sentence has to be segmented into multiple single aspect reviews because people often express differing opinions on multiple aspects simultaneously in the same review, Efficiently poll opinions [4] to discover customer satisfaction by determining whether the comments are positive, negative or neutral.

\section{Literature Survey}

Turney (2002) [1] presented a simple unsupervised learning algorithm to classify the reviews based on recommended (thumbs up) or not recommended (thumbs down) reviews online. The sentiment classification of a review is predicted by the average semantic orientation (SO) of adjective or adverb phrases in the review. Opinions are usually expressed by adjectives and adverbs. They used Point-wise Mutual Information (PMI) and Information Retrieval (IR) to measure the similarity of pairs of words or phrases, which is to calculate semantic orientation ( $\mathrm{SO}$ ) of a word or phrase by subtracting mutual information between the word or phrase and the reference word excellent from the mutual information between the word or phrase and the reference word poor. The mutual information is the co-occurrence of the two words or phrase among millions of online documents.

Bo Pang (2002) [2] conducted a study on sentiment analysis using movie review data. It was a document-level supervised learning and they applied SVM, Nave Bayesian, and Maximum Entropy to the feature spaces they constructed. They chose several tokens such as n-grams, POS tags, and adjectives as features to feature spaces.

$\mathrm{Hu}$ and Liu (2004) [3] proposed a system to use association rule mining to extract frequent noun phrases as potential product features. In the second step, all adjectives that were treated as potential opinion words in sentences that contained product features were extracted. Then, for each product feature in the sentence, the nearby adjective was treated as its effective opinion. In the third step, the polarities (positive or negative) of opinion words were decided using WordNet and bootstrapping methods.

Seyed Hamid Ghorashi1, Roliana Ibrahim2*, Shirin 


\section{International Journal of Science and Research (IJSR) \\ ISSN (Online): 2319-7064}

Index Copernicus Value (2013): 6.14 | Impact Factor (2014): 5.611

Noekhah3 and Niloufar Salehi Dastjerdi4(2012)[5] This work consists of five phases:1) preprocessing 2)POS 3) Frequent Feature Identification:-H mine is used in place of Apriori Algorithm to find frequent item sets. The minimum support value of the algorithm is set to 1 percent meaning that all the patterns that can be found in at least 1 percent ofthe review sentences are considered as frequent features.4) Pruning-compact feature are a feature phrase that its words do not appear together in the sentence. At least one occurrence of the two words which appear in a sentence with distance of 3 . If it cannot find a sentence, the feature will be removed from the list.

Edison Marrese-Taylor, Juan D. Vel asquez, Felipe BravoMarquez, Yutaka Matsuo (2013) [6] They proposed models to define and extract opinions from web documents. However, the algorithm for aspect expressions extraction, based on frequent nouns and NPs appearing in reviews, achieved a poor performance in the tourism domain. Results show that, in fact, multiple expressions are used to denote the same attribute or component of a tourism product in reviews. Therefore, not only the most frequent words need to be considered when extracting aspect expressions in order to achieve a better recall for this task.

\section{Problem Definition}

Supervised classification algorithms [10] [11] were used to predict the polarities of user reviews [2]. However, several challenges exist for these approaches. The existing approach uses supervised learning algorithm which needs labeling the data that is often expensive and time consuming. It is desirable to develop a learning algorithm that does not require large amounts of labelled training data. Second, traditional document-level classification techniques do not always originate meaningful aspect-based opinion polling. Also it couldn't satisfactorily deal with the implicit aspect expression problem which remains to be a challenge in opinion mining domain.

People tend to express multiple opinions on multiple aspects in a single review and in a single sentence. This sentence is called multi-aspect sentence. Therefore, handling a multiaspect sentence as a single-aspect would not lead to satisfactory results in identifying customer opinion effectively. This raises an important and practical question of how to segment a multi-aspect sentence into multiple single-aspect units, referred to as aspect-based sentence segmentation.

\section{Project Scope}

The project will be limited to extracting aspects from customer reviews of a particular product. The system will extract both explicit aspects as well as implicit aspects. Finally, a summary of customer reviews referring to the user queried aspect, based on its sentiment polarity will be displayed. The project is divided into four modules based on its functionality. They are: - Aspect extraction, Aspect Reduction, Rule Generation, Summarization.

\section{Implementation Strategy}

To analyze textual reviews, some previous studies attempted to predict the polarities of these user reviews by using supervised document classification algorithms [2]. Some recent work has expanded polarity analysis on a multipoint scale under ranking or ordinal regression frameworks in the fashion of supervised learning. It couldn't satisfactorily deal with the implicit aspect expression problem that occurs frequently. Aspect-based opinion polling lies where people often express differing opinions on multiple aspects simultaneously in the same review and even in the same sentence; this is called a multi-aspect sentence. Therefore, treating a multi-aspect sentence as a single-aspect mention for aspect-based opinion polling would not lead to satisfactory results. This raises an important and practical question of how to segment a multi-aspect sentence into multiple single-aspect units, referred to as aspect-based sentence segmentation.

People tend to express multiple opinions on multiple aspects in a single review and in a single sentence and its general tendency of customers to tell stories about their experiences when writing reviews. The expressed reviews are more credible to have lengthy and more complex sentences, which describes about product features multiple times. Reviewers also usually mention objects that do not correspond to attributes or components of the reviewed product. It is important to note that substantial number of sentences in a review do not contain opinions. These sentences must be excluded while analyzing reviews and processing opinions. Existing approaches do not take a call to address these challenges.

A set of new rules are to be developed in a proposed system that would address the appearance of compound aspects consisting of more than one term and also cover those aspects that tend to appear multiple times in a single sentence. In addition, this project proposes to include Point wise mutual information [3][12] to discover the implicit aspects or emotions hidden in the reviews.

Ultimately this project would permit the discovery of customer preferences when applied to tourism product reviews thus making it domain sensitive. In the case of the tourism industry, the existing study of customer preferences is implemented using traditional tools that fail to cover a significantly representative number of participants because they are applied to specific groups of people. In this context, aspect based opinion mining offers a larger scope method to understand aggregated consumer preferences.

Another important drawback of the existing system is that they are not domain specific. Domain Specific sentences are needed to be treated specifically. In the tourism domain, this could constitute to be a major problem since a lot of opinions could imply a positive or negative sentiment depending on the product on which the opinion is given on. The proposed project will try to resolve this problem by making it domain specific and finding aspect synonyms using PMI [3] 
International Journal of Science and Research (IJSR)

ISSN (Online): 2319-7064

Index Copernicus Value (2013): 6.14 | Impact Factor (2014): 5.611

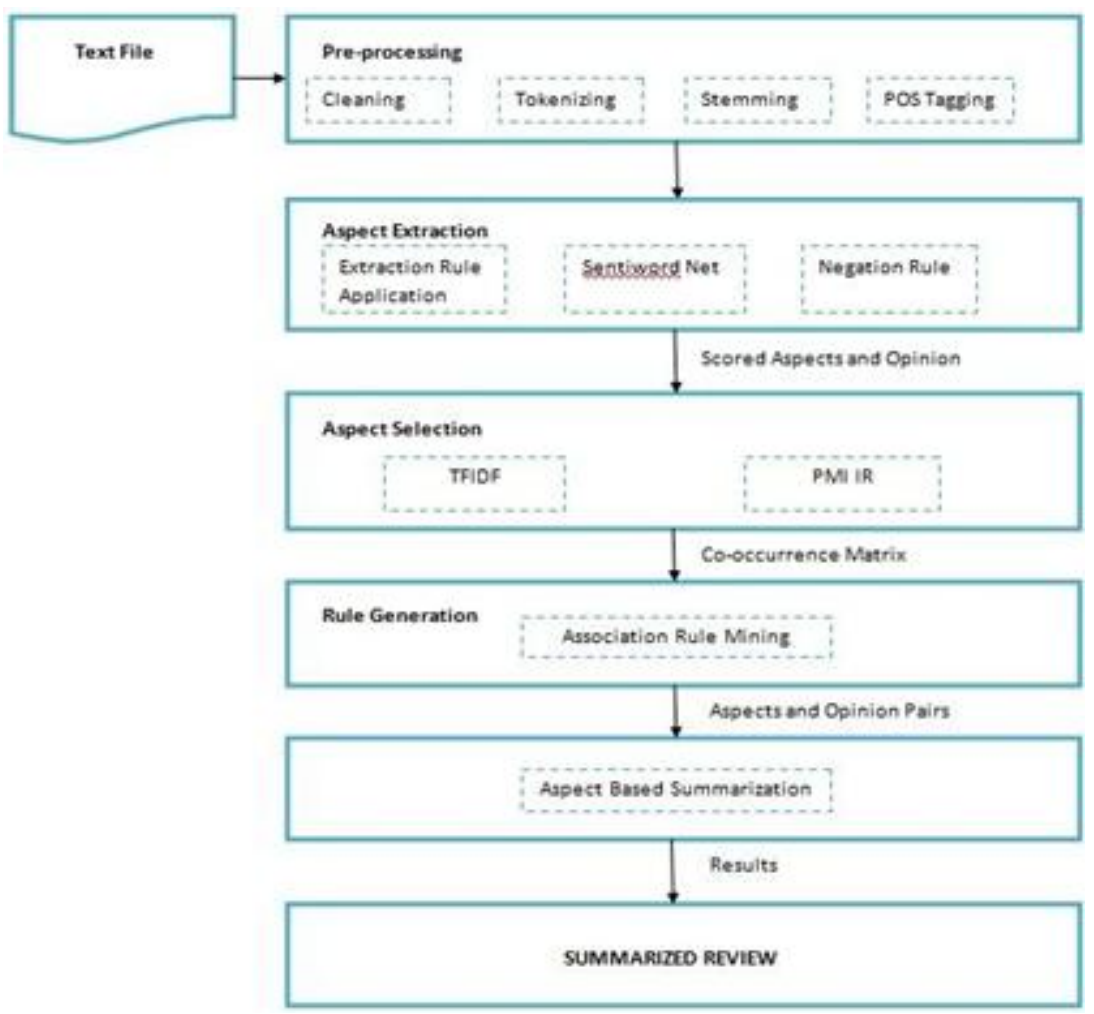

Figure 1: System Architecture

\section{Proposed System}

\section{A. System Architecture}

System architecture shown in diagram. A text file is given as input to pre processing block which passes through various steps such as cleaning, tokenizing, stemming, POS tagging. Aspect extraction is done by applying extraction rule application and negation rule. Aspect selection is done using TFIDF and PMI IR. Aspects and Opinion pairing is done using association rule mining.

\section{B. Algorithm}

Step 1: Aspect Extraction: Part of speech tagger [7] is used for extracting aspects that are usually nouns. Adjectives, Adverbs which represent opinions over aspects are also extracted using the same.

Step 2: SentiWordNet [8][9] is used for finding sentiment polarity. A score is associated with every sentiment expressed.

Step 3: Segmentation: Select aspect based sentences and eliminates irrelevant nouns using PMI IR.

Step 4: Rule Generation: Generating aspect-opinion pairs using co-occurrence association rule mining.

Step 5: Summarization: Aspect based summary on customer reviews using the rules generated.

\section{Mathematical Model}

I is input dataset containing customer reviews about product as i1, i2, i3 ... in.

$I=$ as $i 1, i 2, i 3 \ldots$ in

$\mathrm{S}=\mathrm{s} 1, \mathrm{~s} 2, \mathrm{~s} 3 \ldots \mathrm{sn}$

Where $\mathrm{s} 1, \mathrm{~s} 2, \mathrm{~s} 3 \ldots \mathrm{sn}$ are the review sentences of users that holds opinions about the product.

P1: Data pre-processing in which stemming and other operations are done so as to make data compatible for next round of processing

P2: Classification of the review or comments as aspects and sentiments

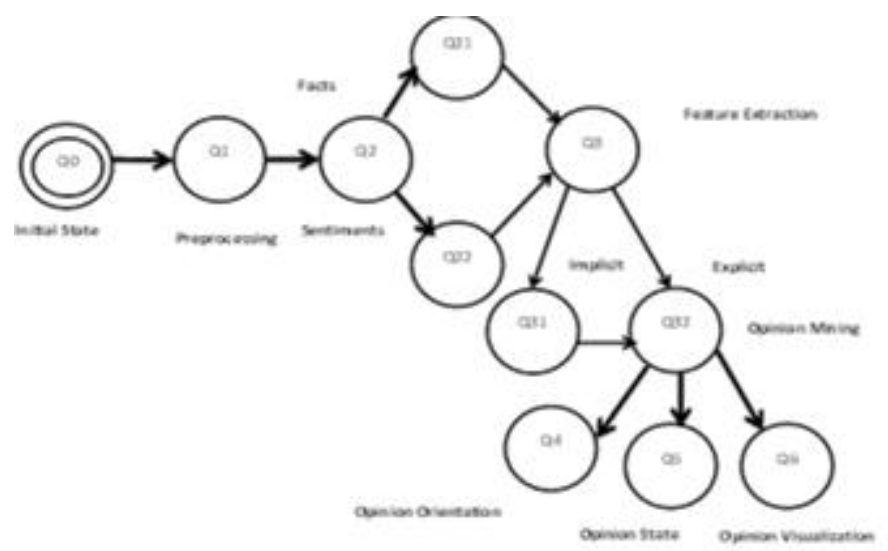

Figure 2: Process State Diagram

P3: Classify the sentiments as implicit opinion and explicit opinion

P4: Feature extraction to extract implicit as well as explicit features of the product 


\section{International Journal of Science and Research (IJSR) \\ ISSN (Online): 2319-7064}

Index Copernicus Value (2013): 6.14 | Impact Factor (2014): 5.611

P5: Opinion orientation to find sentiment polarity

P6: Status indicator of oriented opinion i.e. either the input user review is positive, negative or neutral

P7: Visualization of each opinion status to decision making process

$\mathrm{P}=\mathrm{P} 1, \mathrm{P} 2, \mathrm{P} 3, \mathrm{P} 4, \mathrm{P} 5, \mathrm{P} 6, \mathrm{P} 7$.

The rule serves as a factor to decide the opinion orientation i.e. positive, negative or neutral. Below three rules are defined $\mathrm{R}=\mathrm{R} 1, \mathrm{R} 2, \mathrm{R} 3$

Where, $\mathrm{R} 1=$ If the final score is positive; then opinion on the feature in sentence $\mathrm{S}$ is considered as Positive.

$\mathrm{R} 2=$ if the final score is negative, then opinion on the feature in sentence $\mathrm{S}$ is considered as Negative.

$\mathrm{R} 3=$ otherwise opinion on the feature in sentence $\mathrm{S}$ is Neutral Output $\mathrm{O}=\mathrm{O} 1, \mathrm{O} 2, \mathrm{O} 3$

Where, O1: Positive opinion; O2: Negative opinion; O3: Neutral opinion.

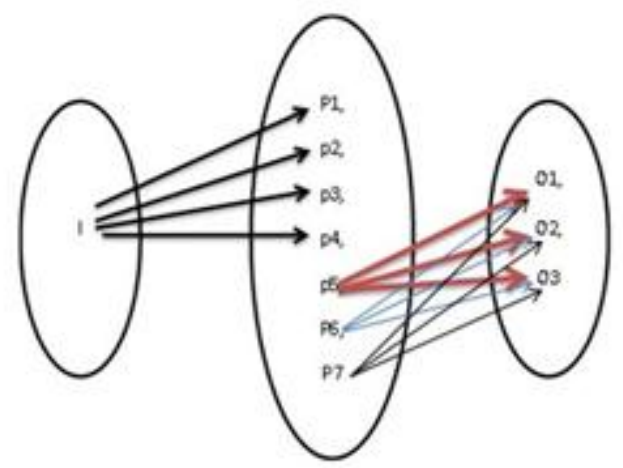

Figure 3: Venn Diagram to represent Mapping between Input, Process and Output of System

\section{Result}

\section{A. Aspect Extraction}

Total of words/ tokens generated from the reviews $=29844$

The percentage reduction after stemming $=25.6 \%$

Table 2: ASPECT EXTRACTION

\begin{tabular}{|c|c|c|c|c|}
\hline $\begin{array}{c}\text { No of } \\
\text { Aspects } \\
\text { Extracted by } \\
\text { POS Tagger }\end{array}$ & $\begin{array}{c}\text { No of Aspects } \\
\text { Correctly } \\
\text { Extracted by } \\
\text { POS Tagger }\end{array}$ & $\begin{array}{c}\text { No of } \\
\text { Aspects Not } \\
\text { Extracted by } \\
\text { POS Tagger }\end{array}$ & Precision & Recall \\
\hline 313 & 210 & 41 & $67.09 \%$ & $83.66 \%$ \\
\hline
\end{tabular}

\section{B. Opinion Extraction}

\begin{tabular}{|c|c|c|c|c|}
\hline $\begin{array}{c}\text { No of } \\
\text { Opinions } \\
\text { Extracted } \\
\text { by POS } \\
\text { Tagger }\end{array}$ & $\begin{array}{c}\text { No of } \\
\text { Opinions } \\
\text { Correctl y } \\
\text { Extracted } \\
\text { by } \\
\text { POS } \\
\text { Tagger }\end{array}$ & $\begin{array}{c}\text { No of } \\
\text { Opinions } \\
\text { Not } \\
\text { Extracte } \\
\text { d by POS } \\
\text { Tagger }\end{array}$ & Precisio n & Recall \\
\hline 188 & 136 & 68 & $72.34 \%$ & $66.67 \%$ \\
\hline
\end{tabular}

This precision drops as a result of extracting many nouns/noun phrases which are not product aspects. The reason is that during extraction, adjectives which are not opinionated will be extracted as opinion words, thus leading to extracting wrong aspects.

\section{Sentiment Polarity}

Table 3: Sentiment Extraction

\begin{tabular}{|c|c|c|}
\hline Polarity & $\begin{array}{c}\text { Positive } \\
\text { Opinion }\end{array}$ & $\begin{array}{c}\text { Negative } \\
\text { Opinion }\end{array}$ \\
\hline $\begin{array}{c}\text { Sentiment Polarity by } \\
\text { SentiWordNet }\end{array}$ & 107 & 64 \\
\hline $\begin{array}{c}\text { Sentiment Polarity Correctly } \\
\text { identified by SenTiWordNet- }\end{array}$ & 79 & 37 \\
\hline Precision & $73.83 \%$ & $57.81 \%$ \\
\hline
\end{tabular}

\section{Aspect Generated}

Table 4: Precision Aspects Generated

\begin{tabular}{|c|c|c|}
\hline $\begin{array}{c}\text { No. of Manual aspects } \\
\text { by Hu et al. }\end{array}$ & $\begin{array}{c}\text { No of aspects correctly } \\
\text { generated by POS }\end{array}$ & Precision \\
\hline 111 & 92 & $82.88 \%$ \\
\hline
\end{tabular}

$\mathrm{Hu}$ et al. (2004) [3] tagged all the aspects on which the reviewer has expressed his/her opinions. If the user gave no opinion in a sentence it was not tagged. Many aspects like "dependable ","compactable" were also found to be aspects. These words are extracted as sentiment words by the proposed model of the project.

\section{E. Accuracy of Sentiment Orientation}

Table 5: Accuracy of Sentiment Orientation

\begin{tabular}{|c|c|}
\hline $\begin{array}{c}\text { Sentiment Orientation accuracy } \\
\text { obtained by Hu, M. \& Liu, B }\end{array}$ & $\begin{array}{c}\text { Sentiment Orientation } \\
\text { Accuracy by SentiWordNet }\end{array}$ \\
\hline $76.4 \%$ & $65 \%$ \\
\hline
\end{tabular}

Table 5 result shows the shortcoming of the method used for assigning sentiment polarity. Assigning the same polarities of the opinion words to the product aspects does not work well in most situations. Hu et al. (2004). [3] After extracting the potential opinion words, they identified the polarities of the opinion words by utilizing synonymous set and antonymous set in the WordNet, and a small list of opinion words with opinion polarities.

The product review corpus was collected from TripAdvisor.in TripAdvisors is the source of reviews, which includes user reviews for tourism products. The corpus contains 300 reviews. Each of the review includes a text review. Additional information available but not used in this project includes date, time, author name, location and ratings. Reviews based on tourism products were manually collected. A typical review contains free text summary about a product. All reviews are plain text. Aspect selection will be done using PMI IR. The point-wise mutual information (PMI) Mi(w) between the word $\mathrm{w}$ and the class $\mathrm{i}$ is defined on the basis of the level of co-occurrence between the class $i$ and word $\mathrm{w}$. The expected co-occurrence of class $\mathrm{i}$ and word $\mathrm{w}$, on the basis of mutual independence, is given by $\mathrm{Pi} F(\mathrm{w})$, and the true co occurrence is given by $\mathrm{F}(\mathrm{w}) \mathrm{pi}(\mathrm{w})$. The mutual information is defined in terms of the ratio between these two values and is given by the following equation: 


\section{International Journal of Science and Research (IJSR)}

ISSN (Online): 2319-7064

Index Copernicus Value (2013): 6.14 | Impact Factor (2014): 5.611

$$
M_{i}(w)=\log \left(\frac{F(w) \cdot p_{i}(w)}{F(w) \cdot P_{i}}\right)=\log \left(\frac{p_{i}(w)}{P_{i}}\right)
$$

\section{Conclusion}

An aspect-based opinion mining technique allows us to analyze opinions about product features or the aspects that are associated with the product such as product components and attributes. This project extracts aspects and the related customer sentiments on tourism domain. This proffer an approach to discover consumer preferences about products and services using statistical opinion mining. The proposed system extracts both explicit aspects as well as implicit aspects from customer reviews thus increasing the sentiment orientation of opinion. Most of the researches were based on extracting explicit opinions of customers. This system tries to retrieve implicit sentiments of customer. Due to the growing availability of unstructured reviews, the proposed system allows summarizing the information hence obtained in order to provide customers with precise results

\section{Future Scope}

It is vital aspect to deal with the issue of fake reviews). Popularly known as Opinion spam is an act of writing fake or bogus reviews in order to deliberately mislead readers by giving fake positive and/or negative opinions. This is done either to promote products and gain popularity in the market or to damage the reputations of some other objects. Another important factor to be considered in the field of opinion mining is to detect sarcasm. It is generally observed that sarcasm and negative opinion are correlated. Another important aspect to deal with is the domain dependency. Words that are used to express opinion in a particular domain may not hold the same polarity in another domain; this must be handled in an appropriate manner.

\section{Acknowledgment}

It is a pleasure for authors to thank all the people who in different ways have supported us in completing this study and contributed to the process of writing this paper.

\section{References}

[1] Turney, p. (2002). Thumbs up or thumbs down? Semantic orientation applied to unsupervised classification of reviews, In Proceedings of the 40th Annual Meeting of the Association for Computational Linguistics, Philadelphia, Pennsylvania.

[2] Pang, B., Lee, L., and Vaithyanathan, S. (2002). Thumbs up? Sentiment Classification Using Machine Learning Techniques, In Proc. of EMNLP.

[3] $\mathrm{Hu}, \mathrm{M}$. and Liu, B. 2004. Mining and summarizing customer reviews. International Conference on Knowledge Discovery and Data Mining (ICDM).

[4] Ding, X., Liu, B. and Yu, P. S. (2008). A holistic lexicon-based approach to opinion mining, In Proceedings of the Conference on Web Search and Web Data Mining (WSDM).

[5] Seyed Hamid Ghorashi1, Roliana Ibrahim2*, Shirin
Noekhah3 and Niloufar Salehi Dastjerdi4(2012). A Frequent Pattern Mining Algorithm for Feature Extraction of Customer Reviews, Procedia IJCSI, Vol 9, Issue 4, No 1

[6] Edison Marrese-Taylor, Juan D. Vel asquez, Felipe Bravo-Marquez, Yutaka Matsuo (2013). Identifying Customer Preferences about Tourism Products using an Aspect-Based Opinion Mining Approach, Procedia Computer Science 22 ( 2013 ) 182 191,Elsevier

[7] Kristina Toutanova, Dan Klein, Christopher Manning, and Yoram Singer. 2003. Feature-Rich Part-of-Speech Tagging with a Cyclic Dependency Network. In Proceedings of HLT-NAACL 2003, pp. 252-259.

[8] Zhu J.,H.Wang,M,Zhu and B.K.Tsou.2011.Aspect based opinion polling from customer reviews. IEEE Transactions on Affective Computing,2(1):37-49.37

[9] Bo Pang and Lillian Lee. 2004. A sentimental education: Sentiment analysis using subjectivity summarization based on minimum cuts. In Proceedings of ACL-04, 42nd Meeting of the Association for Computational Linguistics, pages 271278, Barcelona, ES.

[10]Isabella, J Analysis and evaluation of Feature selectors in opinion mining, Indian Journal of Computer Science and Engineering (IJCSE), Vol. 3 No.6 Dec 2012-Jan 2013

[11]] Jin, W. and H. Ho. Opinion Miner: a novel machine learning system for web opinion mining and extraction. In Proceedings of ACM SIGKDD International Conference on Knowledge Discovery and Data Mining (KDD-2009), 2009b.

[12]Qi Su,Kun Xiang, Houfeng Wang, Bin Sun and Shiwen $\mathrm{Yu}(2006)$. Using Pointwise Mutual Information to Identify Implicit Features in Customer Reviews. ICCPOL,LNAI 4285,pp.22-30,Springer(2006). 